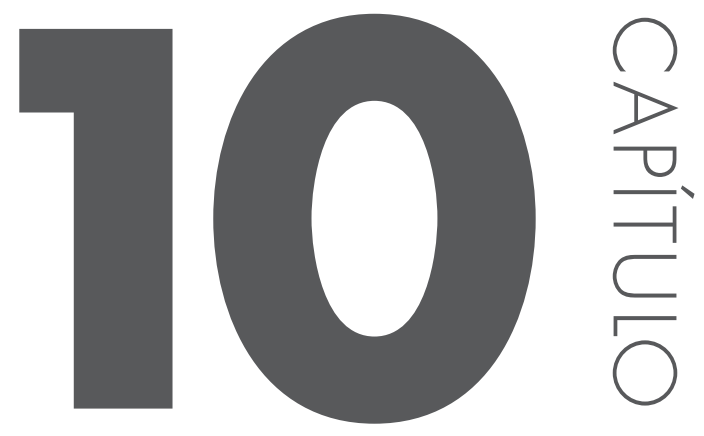

\title{
CONTAMINANTES EMERGENTES: REMOVENDO ESTES POLUENTES DO MEIO AQUÁTICO
}

Sousa, Priscila Afonso Rodrigues 1 *;

Coelho, Luciana Melo ${ }^{1}$

1 Universidade Federal de Goiás/Regional Catalão. Av. Dr. Lamartine Pinto de Avelar, 1 120, Setor Universitário, Catalão, Goiás, Brasil.

* email: rodriguessousa41 @ hotmail.com 
Resumo: Dentre os diversos contaminantes dos recursos hídricos destacamse os contaminantes emergentes que são substâncias presentes no meio ambiente há anos devido ao descarte indiscriminado de esgoto e efluentes. Neste sentido, técnicas estão sendo desenvolvidas para promover a remoção destes compostos do ambiente aquático. $E$, dentre as técnicas recentemente apresentadas pela literatura, tem-se em destaque o uso de bioadsorventes para esta finalidade. No presente estudo serão mostrados resultados da utilização da casca de banana como bioadsorvente para remoção de contaminante emergente de amostras de água. Deste estudo observaram-se resultados satisfatórios com a aplicação deste bioadsorvente que apresentou índices de remoção superior a $80 \%$. Resultado este que ilustra a aplicabilidade de tal sistema para utilização em processos de remoção de polventes no meio aquático.

\section{Palavras-chave: Bioadsorvente; Contaminante; Remoção}




\section{Introdução}

A água, enquanto recurso necessário para a sobrevivência de todos os seres vivos deve ser preservada e utilizada de maneira correta. Contudo, o que se tem observado são ameaças advindas do uso inadequado e da poluição, seja de ordem natural ou promovida pelo homem.

Segundo Collins (2011) dentre os produtos considerados poluentes encontram-se os contaminantes emergentes os quais compreendem produtos farmacêuticos e de higiene pessoal (PFHP), subprodutos industriais, hormônios naturais e drogas ilícitas, os quais são indicadores de atividade antrópica. 
De acordo com a Organização Mundial de saúde (OMS), a concentração dos inúmeros contaminantes naturais e sintéticos nas águas subterrâneas ou superficiais se dá na faixa de $\mathrm{pg} \mathrm{L}^{-1}$ a $\mathrm{ng} \mathrm{L}^{-1}$. Apesar de serem encontrados em concentração relativamente baixa, tais compostos representam um risco à saúde uma vez que ocasionam modificações no organismo dos seres devido aos processos de bioacumulação.

Dentre os contaminantes emergentes, destacam-se os desreguladores endócrinos presentes em medicamentos para regulação hormonal ou utilizado como contraceptivo.

\subsection{Desreguladores Endócrinos (DE)}

Os desreguladores ou interferentes endócrinos fazem parte de uma categoria recente de polventes ambientais que atuam e interferem nas várias funções do sistema endócrino, dentre estes se pode destacar os hormônios e substancias sintéticas ou naturais.

A necessidade em estudar tais substâncias deve-se ao fato das mesmas ocasionarem diversos efeitos sobre os organismos que entram em contato com as mesmas. Dentre estes efeitos pode-se destacar: feminização de peixes machos; alterações no sistema imunológico de mamíferos, alterações irreversíveis no sistema reprodutivo em peixes, répteis e fauna em geral. Em seres humanos cita-se a redução da quantidade de esperma, o aumento da incidência de câncer de mama e incidência de endometriose.

Várias são as definições utilizadas para destacar o que seria um desregulador endócrino. Segundo o Programa Internacional de Segurança Química (IPCS), "Um desregulador endócrino é uma substância ou um composto exógeno que altera uma ou várias funções do sistema endócrino e tem, consequentemente, efeitos adversos sobre a saúde num organismo intacto, sua descendência, ou (sub) populações".

$\mathrm{Na}$ tentativa de buscar alternativas quanto a polvição ocasionada pelos desreguladores endócrinos várias organizações tais como a Agência de Proteção Ambiental dos Estados Unidos (EPA), União Européia (UE), Organização Mundial de Saúde (OMS), Programa Internacional de Segurança Química (IPCS), dentre outras, realizam estudos e monitoramentos em várias regiões, na tentativa de compreender a ação que tais substancias exercem sobre os diferentes seres.

De acordo com Dezotti (2007) as diversas substâncias classificadas como desreguladores endócrinos podem ser divididas nos seguintes grupos:

1. Substâncias sintéticas - utilizadas na agricultura e seus subprodutos, como pesticidas, herbicidas, fungicidas e moluscicidas; utilizadas nas indústrias e seus subprodutos, como dioxinas, PCB, alquilfenóis e seus subprodutos, HAP, ftalatos, bisfenol $A$, metais pesados, entre outros; compostos farmacêuticos, como os estrogênios sintéticos $17 \alpha$ etinilestradiol;

2. Substâncias naturais - fitoestrogênios, tais como, genisteína e metaresinol e estrogênios naturais $17 \beta$-estradiol, estrona e estriol.

Dentre os desreguladores endócrinos existentes destacam-se os estrogênios endógenos, os resíduos de pesticidas, tais como herbicidas e fungicidas, os quais vêm sendo encontrados 
em alimentos, água potável e corpos hídricos. Na indústria química, substâncias como detergentes, resinas, alguns aditivos, monômeros, ftalatos, dentre outros utilizados na produção de plásticos também são incluídos.

Dentre os estrogênios endógenos, destaca-se os produzidos naturalmente pelo corpo humano, como por ex., o 17ß-estradiol e a estrona, além de outros sintéticos e utilizados em produtos farmacêuticos como o 17a-etinilestradiol (Figura 1).

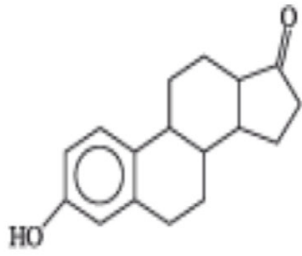

Estrona

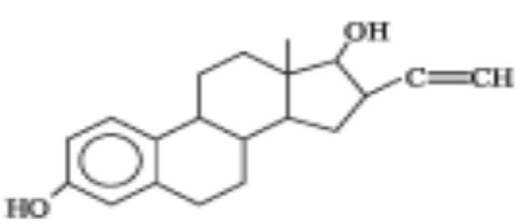

$17 \alpha$-Etinilestradiol

Figura 1: Estrogênios endógenos (desreguladores endócrinos)

Entre os estrogênios endógenos estudados neste trabalho, destacam-se os hormônios estrona e $17 \alpha$-etinilestradiol. O hormônio estrona (E1) é um derivado do $17 \beta$-estradiol sendo sintetizado e secretado pelo ovário predominante nas mulheres principalmente após a menopausa. Este hormônio é secretado pelas células de gordura e durante a gravidez, pela placenta. Todos vertebrados do sexo feminino, e em menor quantidade o sexo masculino produzem certa quantidade de estrona, sendo que esta substância encontra-se presente no meio ambiente por meio da excreção (GUIMARÃES, 2008).

O $17 \alpha$-etinilestradiol (EE2) é sintetizado artificialmente possuindo características físicoquímicas semelhantes com as dos hormônios naturais. É um derivado do estrogênio $17 \beta-$ estradiol, sendo encontrado em pílulas anticoncepcionais e pílulas do dia seguinte, é utilizado por via oral e liberado para o meio ambiente a partir da urina e fezes (GUIMARÃES, 2008). As principais características da estrona e do $17 \alpha$-etinilestradiol estão destacados na Tabela 1.

Tabela 1: Principais características físico-químicas do $17 \alpha$-etinilestradiol, e da estrona.

\begin{tabular}{|l|l|l}
\hline Substância & Estrona & $\mathbf{1 7} \alpha$-etinilestradiol \\
\hline Sigla & El & EE2 \\
\hline Massa Molecular $(\mathrm{g} / \mathrm{mol})$ & 270,0 & 296,4 \\
\hline Solubilidade em água $(\mathrm{mg} / \mathrm{L})$ & 13,0 & 4,8 \\
\hline pKa $\left(20^{\circ} \mathrm{C}\right)$ & 10,4 & 10,4 \\
\hline log Kow & 3,1 & 3,6 \\
\hline Pressão de Vapor $(\mathrm{mmHg})$ & $2,3 \times 10^{-10}$ & $4,5 \times 10^{-11}$ \\
\hline
\end{tabular}

(Ghiselli e Jardim, 2007; Yangali-Quintanilla et al., 2009). 
Devido à baixa pressão de vapor dos compostos, pode-se dizer que os mesmos possuem baixa volatilidade, sendo assim à opção pela determinação em Cromatografia Liquida de Alta Eficiência, mostra-se mais adequada, se comparada à determinação por Cromatografia Gasosa, visto que seriam necessárias temperaturas altas para propiciar volatilização dos compostos estudados, o que poderia acarretar danos à coluna cromatográfica.

Sendo assim este trabalho realiza o estudo da capacidade do adsorvente casca de banana na remoção do composto estrona presentes em matrizes aquosas através de processos de adsorção em que moléculas no estado líquido são colocadas em contato com um composto sólido na tentativa de promover interações e reter uma ou mais espécies sobre a superfície sólida (ALBERS, 2002).

\section{Objetivos}

O presente trabalho tem como objetivos: Preparar e caracterizar a casca da banana para aplicação como bioadsorvente na remoção de compostos orgânicos, assim como propor uma metodologia para determinação de etinilestradiol e estrona em água utilizando sistema HPLC.

\section{Metodologia}

\subsection{Caracterização por Espectroscopia na região do infravermelho (FTIR)}

Para realização das devidas caracterizações por FTIR realizou-se o registro dos espectros do adsorvente na região do infravermelho médio utilizando um Espectrofotômetro de Infravermelho Modelo IR. Prestige - 21. Para isso, secaram-se as amostras e prensaram na forma de pastilhas de $\mathrm{KBr}$ na proporção 100:1 KBr/amostra. A análise foi realizada na faixa de número de ondas entre 4000 e $500 \mathrm{~cm}^{-1}$, com resolução de $4 \mathrm{~cm}^{-1}$.

\subsection{Preparação dos adsorventes:}

As cascas de banana foram secas em estufa, trituradas e separadas na granulometria de 100 mesh. Cerca de $10 \mathrm{~g}$ do adsorvente foi agitado por $60 \mathrm{~min}$ com 250 $\mathrm{mL}$ de água deionizada, filtrado e seco em estufa a $75{ }^{\circ} \mathrm{C}$ por $24 \mathrm{~h}$, este material é denominado de adsorvente "in natura". O mesmo procedimento foi realizado substituindo a água deionizada por hidróxido de sódio $\left(0,1 \mathrm{~mol}^{-1}\right)$ e ácido clorídrico $(0,1$ mol $\left.L^{-1}\right)$ e o material final denominado adsorvente tratado com $\mathrm{NaOH}$ e $\mathrm{HCl}$, 
respectivamente. A avaliação do tratamento do material adsorvente foram feitos em erlenmeyer de $250 \mathrm{~mL}$, contendo $500 \mathrm{mg}$ do material adsorvente (in natura, tratado com $\mathrm{NaOH}$ e com HCl) e $25,0 \mathrm{~mL}$ de solução contendo $1,0 \mathrm{mg} / \mathrm{L}$ das respectivas substâncias estudadas $(17 \alpha-$ etinilestradiol e estrona) e mantidos sob agitação à temperatura ambiente durante 60 minutos. A solução foi então filtrada e a leitura da solução final foi realizada por sistema HPLC (do inglês High Performance/Pressure Liquide Chromatography).

\section{Resulfados e Discussão}

\subsection{Caracterização do Bioadsorvente: Espectroscopia da Região do Infravermelho Médio}

Os grupos funcionais ativos nos adsorventes são de fundamental importância para os processos de interação entre adsorvente e adsorbato. O espectro obtido na região do infravermelho médio (FT-MIR) mostram os grupos funcionais presentes no adsorvente estudado. O espectro de Infravermelho Médio da casca da banana in natura mostra a presença de algumas bandas típicas de alguns grupos funcionas, tais como hidroxila, ésteres, aldeídos e amidas (Figura 2).

De acordo com o espectro do adsorvente in natura observam-se diversas bandas. A banda larga centrada em aproximadamente $3400 \mathrm{~cm}^{-1}$ caracteriza vibrações em grupos hidroxila, provenientes da água absorvida na superfície do material. Foi possível identificar também a presença de metilcelulose através da detecção de v (C-H) e $\delta$ (C-H) em bandas de absorção localizadas no espectro em $2927 \mathrm{~cm}^{-1}$ (GONDIM et al., 2005).

O pico observado em $1625 \mathrm{~cm}^{-1}$ é atribuído a carbonila de amidas primárias presentes na porção protéica. A bandas centrada em $1059 \mathrm{~cm}^{-1}$ pertencem à vibração superior v (C-O) de álcool primário e secundário respectivamente (VERSIANI, 2008). A banda em $1750 \mathrm{~cm}^{-1}$ representa o estiramento $\mathrm{C}=\mathrm{O}$ indicando a presença de éster. 


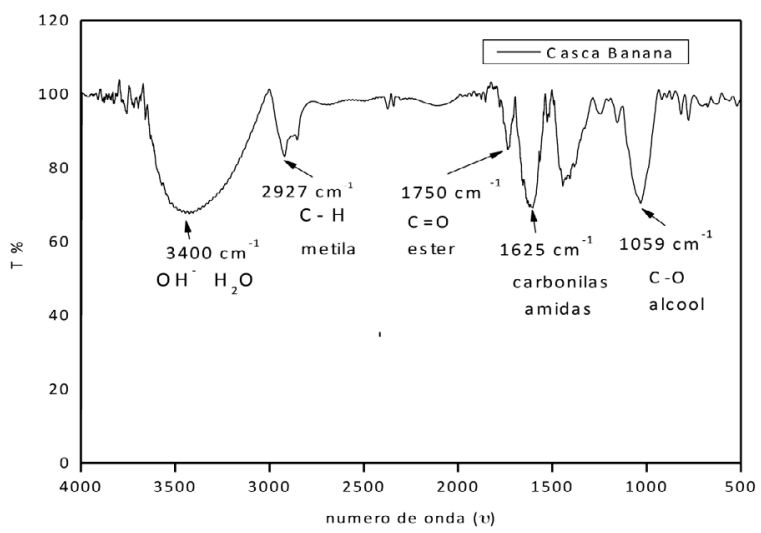

Figura 2 : Espectro de Infravermelho Médio da Casca de Banana in natura.

\subsection{Validação}

Na Tabela 2 são apresentados os resultados relativos aos parâmetros de mérito até então estudados para o método de separação e detecção avaliado (HPLC).

Tabela 2: Parâmetros de Mérito

\begin{tabular}{|c|c|c|c|c|c|}
\hline Composto & Tempo & Linearidade/r & DPR & LD & $L Q$ \\
\hline & retençāo (min) & $2_{1}$ & $(\%)$ & $\left(\mathrm{mg}^{-1} \mathrm{~L}^{-1}\right)$ & $\left(\mathrm{mg} \mathrm{L}^{-1}\right)$ \\
\hline El & 5,200 & 0,998 & 6,640 & 0,025 & 0,050 \\
\hline EE2 & 4,600 & 0,999 & 4,250 & 0,030 & 0,050 \\
\hline
\end{tabular}

aDPR: Desvio Padrão Relativo; bLD: Limite de Detecção; ${ }^{2} L Q$ : Limite de Quantificação.

Os estudos de adsorção dos hormônios $17 \alpha$ - etinilestradiol e estrona foram realizados utilizando o bioadsorvente "in natura", tratado com $\mathrm{HCl}$ e $\mathrm{NaOH}$. Pela figura 3 observa-se que o maior índice de remoção dos compostos foi com o bioadsorvente tratado com $\mathrm{NaOH}$. Isto ocorre porque o hidróxido de sódio consegue remover taninos presentes no bioadsorvente. Além disso, o tratamento tanto com $\mathrm{HCl}$ quanto com $\mathrm{NaOH}$ promoveu a modificação dos grupos funcionais presentes na superfície do bioadsorvente. Com a criação de novos sítios ativos há maior interação com os compostos em estudo, permitindo maior adsorção dos mesmos, situação esta que não ocorre com o material "in natura". Ressalta-se que apesar de existirem modificações ocasionadas pelo tratamento ácido, as mesmas não são tão eficientes quando comparadas ao tratamento em meio básico, no qual observou-se um aumento intenso da remoção mesmo com a modificação do pH do meio. 
E1

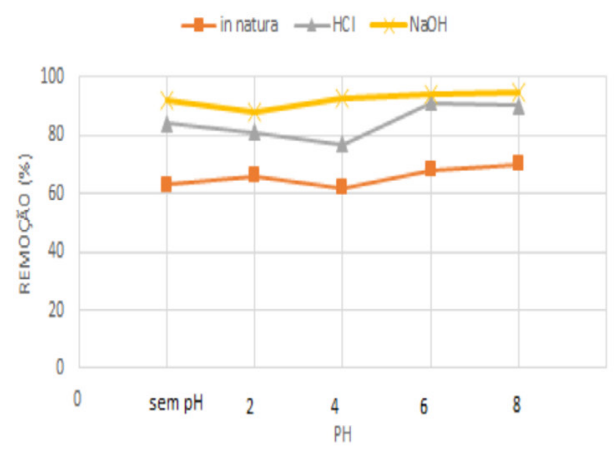

EE2

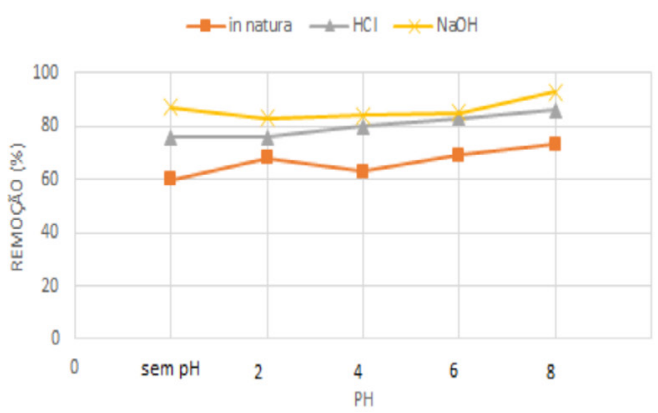

Figura 3: Gráfico de remoção dos compostos E1 e EE2.

Pelos resultados obtidos pode-se concluir que o bioadsorvente é adequado para o uso em processos de adsorção. Ressalta-se que apesar dos compostos serem encontrados na ordem de $\mu^{-1}$ no ambiente aquático, o método utilizado apresenta-se satisfatório uma vez que se observa um bom índice de remoção utilizando a concentração de $1 \mathrm{mg} / \mathrm{L}$, o que permite concluir que a probabilidade destes compostos serem removidos do ambiente aquático, em concentrações menores, também será significativa. 
Contaminants Emerging: removing these pollutants of aquatic environment.

\begin{abstract}
Among the various contaminants of water resources highlight emerging contaminants are substances present in the environment for years due to indiscriminate disposal of sewage and effluents. In this sense, techniques are being developed to promote the removal of these compounds in aquatic environment. Among the newer techniques we highlight the use of bioadsorventes. Therefore, we studied the as bioadsorbent banana peel for removal of contaminant emerging in water samples, the studies proved being satisfactory with indexes removal greater than $80 \%$.
\end{abstract}

\title{
Keywords: Bioadsorbent; Contaminant; Removal.
}

\section{Referências bibliográficas}

ALBERS, A.P.F.; MELCHIADES, F.G.; MACHADO, R.; BALDO, J.B.; BOSCHI, A. O. Um método simples de caracterização de argilominerais por difração de raios X. Cerâmica, vol. 48 (305), p. 34-37, 2002.

COLLINS, C.H., SILVA, C. G. A. Aplicações de Cromatografia Liquida de Alta Eficiência para - Estudo de Poluentes Orgânicos Emergentes. Instituto de Química, Universidade Estadual de Campinas. Quim. Nova, vol. 34, No. 4, 665-676, 2011.

DEZOTTI, M., BILA, D.M. Desreguladores endócrinos no meio ambiente: efeitos e consequências. Quím. Nova, vol.30 n.3 São Paulo maio/jun. 2007.

GHISELLI, G., JARDIM, W. F. Interferentes Endócrinos no Ambiente. Instituto de Química. Universidade Estadual de Campinas. Quim. Nova, vol. 30, No. 3, 2007.

GONDIM, J. A.M.; MOURA, M.F.V.; DANTAS, A.S.; MEDEIROS, R.L. S.; SANTOS, K.M. Composição centesimal e de minerais em cascas de frutas. Ciência e Tecnologia de Alimentos, Campinas, vol. 25, p. 825-827, 2005. 
GUIMARÃES, T.S. Detecção e Quantificação dos hormônios sexuais 17 ß- estradiol (E2), estriol (E3), estrona (E1) e 17 a-etiniestradiol (EE2) em água de abastecimento: estudo de caso da cidade de São Carlos, com vistas ao saneamento ambiental. 2008. 81 f. Dissertação (Mestrado em Química). Instituto de Química, Universidade Federal de São Carlos, São Carlos. 2008.

VERSIANI, L.C.F. Caracterização das propriedades coagulantes e adsorventes de ions Cd (II) em soluções aquosas apresentadas por biomateriais derivados da semente Moringa oleífera. 2008. 109 f. Dissertação (Mestrado em Engenharia Ambiental). Universidade Federal de Ouro Preto, Ouro Preto. 2008.

Yangali-Quintanilla, V., Sadmani, A., McConville, M., Kennedy, M., Amy, G. (2009) "Rejection of pharmaceutically active compounds and endocrine disrupting compounds by clean and fouled nanofiltration membranes." Water Research, vol. 43, 2349-2392. 